\title{
An Integrated Energy-Efficient Capacitive Sensor Digital Interface Circuit
}

\author{
Hesham Omran*, Muhammad Arsalan, Khaled N. Salama \\ Sensors Lab, Electrical Engineering Program \\ King Abdullah University of Science and Technology (KAUST) \\ Thuwal, Kingdom of Saudi Arabia, 23955-6900
}

\section{Abstract}

In this paper, we propose an energy-efficient 13-bit capacitive sensor interface circuit. The proposed design fully relies on successive approximation algorithm, which eliminates the need for oversampling and digital decimation filtering, and thus low-power consumption is achieved. The proposed architecture employs a charge amplifier stage to acheive parasitic insensitive operation and fine absolute resolution. Moreover, the output code is not affected by offset voltages or charge injection. The successive approximation algorithm is implemented in the capacitance-domain using a coarse-fine programmable capacitor array, which allows digitizing wide capacitance range in compact area. Analysis for the maximum achievable resolution due to mismatch is provided. The proposed design is insensitive to any reference voltage or current which translates to low temperature sensitivity. The operation of a prototype fabricated in a standard CMOS technology is experimentally verified using both on-chip and off-chip capacitive sensors. Compared to similar prior work, the fabricated prototype achieves and excellent energy efficiency of $34 \mathrm{pJ} / \mathrm{step}$.

Keywords: Capacitive sensor interface circuit; Capacitance-to-digital conversion (CDC); Low-power; Energy-efficient; CMOS

\footnotetext{
${ }^{*}$ Corresponding author at: King Abdullah University of Science and Technology (KAUST), Thuwal, Saudi Arabia, 23955-6900

Email address: hesham.omran@kaust.edu.sa (Hesham Omran)
} 


\section{Introduction}

Capacitive sensors find numerous applications in the consumer, medical, automotive, and industrial sectors [1]. The range of applications includes, but is not limited to, pressure sensors [2, 3], humidity sensors [4], tactile sensors [5], biological sensing microsystems

5 [6], and chemical detection of volatile organic compounds (VOCs) [7] which can be used as biomarkers for early non-invasive detection of lung cancer [8]. Although the type of application imposes different performance requirements on the sensor interface circuit, energy efficiency is always desirable.

Recent trends in capacitive sensing interface circuits favor direct digitization of the sensor capacitance, rather than performing capacitance to voltage conversion and then digitizing the output voltage $[9,10,11,12]$. Direct digitization offers less complexity, smaller area, and lower power consumption $[9,10,11,12]$. In a "semi-digital" approach, the capacitance can be used to modulate the period or the pulse width of a digital signal $[9,12]$. However, this approach requires a time-to-digital converter in order to provide digital output code, e.g., a fast digital counter and a stable high frequency oscillator [12], which hinders its use in a low-power application. A more attractive approach for capacitance-to-digital conversion (CDC) is the use of $\Delta \Sigma$ modulators [10,11, 13]. However, oversampling and digital decimation filtering required in $\Delta \Sigma$ architectures translate to large power consumption. In addition, $\Delta \Sigma$ interfaces suffer from limited capacitance range to avoid modulator overload $[10,11]$. In order to increase the capacitance range of $\Delta \Sigma$ interface circuits, successive approximation register (SAR) algorithm was proposed to adjust the modulator reference capacitor $[10,11]$. However, the SAR step was only used for initial coarse calibration, while sensor digitization is still performed using the $\Delta \Sigma$ modulator.

A capacitance-to-digital converter (CDC) architecture that fully relies on SAR algorithm will eliminate the need for oversampling, which will reduce power consumption and relax the requirements on the analog blocks. Moreover, digital output code is directly provided with no digital filtering required, which further reduces power consumption. A CDC that fully relies on SAR algorithm was proposed in [14], using an op-amp-less architecture. However, due 
to the absence of the op-amp, the sensor capacitance is connected to a high impedance node which leads to parasitic sensitive design. Moreover, as no charge amplifier stage is present, the change in voltage $(\Delta V)$ that needs to be resolved by the comparator will be inversely proportional to the total sum of the capacitances of the parasitic capacitors, the capacitive sensor, and the SAR capacitor array, which will result in poor absolute resolution (more than $60 f F$ for the design in [14]), in addition to sensitivity to noise, charge injection, and offset voltage. When the CDC circuit is connected to off-chip capacitive sensor, the parasitic capacitor can be very large leading to degradation of the circuit performance. Furthermore, connecting the CDC circuit to off-chip capacitive sensor using a high impedance node makes it highly susceptible to noise coupling.

In this work, an integrated 13 - bit SAR CDC that addresses the previous limitations while maintaining excellent energy efficiency is presented. The proposed CDC employs a charge amplifier stage, which results in insensitivity to parasitic capacitors, insensitivity to charge injection and offset voltages, fine absolute resolution, and immunity to noise coupling. The SAR algorithm is performed in the capacitance-domain using a coarse-fine programmable capacitor array (PCA), which enables digitizing a wide range of capacitance in a compact area. The proposed CDC is insensitive to the value of any reference voltage or current, which translates to very small temperature sensitivity. As the circuit operation is insensitive to parasitic capacitances, it can be used robustly with on-chip and off-chip capacitive sensors and multiplexed capacitive sensor arrays.

The proposed SAR CDC is implemented in a $0.35 \mu \mathrm{m}$ standard CMOS technology. The prototype is capable of digitizing $16 \mathrm{pF}$ capacitance range with $2.75 \mathrm{fF}$ resolution, while occupying only $0.07 \mathrm{~mm}^{2}$ of the chip area. The operation of the fabricated prototype was experimentally verified by using both on-chip and off-chip capacitive sensors. The achieved energy efficiency is $34 \mathrm{pJ} / \mathrm{step}$ which is better than recently published capacitance-to-time and $\Delta \Sigma$ CDCs that are implemented using the same technology and supply voltage [10, $12]$, which shows the merit of the proposed architecture.

The rest of the paper is organized as follows. The operation of the proposed circuit is dis- 
cussed in Section 2, in addition to analysis of the circuit nonidealities. Section 3 describes the programmable capacitor array (PCA). The limits of the PCA resolution and dynamic range due to mismatch are analyzed and calculated. System description and the operation of the digital interface are given in Section 4. Experimental results of the fabricated prototype are presented in Section 5.

\section{Successive Approximation CDC}

\subsection{Circuit Operation}

The schematic of the proposed SAR CDC circuit is shown in Fig. 1. $C_{S E N S}$ is the unknown capacitive sensor and $C_{R E F}$ is a reference capacitor implemented as a binary weighted programmable capacitor array (PCA). $U 1$ is a two-stage Miller compensated op-amp with a PMOS input unbalanced differential pair and $U 2$ is a comparator. The circuit operation is divided into two phases; the precharge phase and the evaluate phase. During the precharge phase $(C L K=1, C L K B=0)$, the op-amp $(U 1)$ is working as a unity gain buffer, i.e., $V_{X}=V_{R E F}$. The charge on the sensing capacitor $\left(C_{S E N S}\right)$ is given by

$$
Q=C_{S E N S} \times V_{R E F}
$$

Next, in the evaluate phase $(C L K=0, C L K B=1)$, the voltage difference across $C_{S E N S}$ is zero, and the charge redistributes to the reference capacitor $\left(C_{R E F}\right)$ and the feedback capacitor $\left(C_{F}\right)$. The charge is given by

$$
Q=C_{R E F} \times V_{R E F}+\left(V_{R E F}-V_{o}\right) \times C_{F}
$$

As the charge is conserved, from (1) and (2), the output of the op-amp is given by

$$
V_{o}=V_{R E F}+V_{R E F} \times\left(\frac{C_{R E F}-C_{S E N S}}{C_{F}}\right) .
$$


75 Thus, the differential input of the comparator (U2) is given by

$$
\Delta V_{o}=V_{o}-V_{R E F}=V_{R E F} \times\left(\frac{C_{R E F}-C_{S E N S}}{C_{F}}\right),
$$

and the output of the comparator is given by

$$
V_{C M P}=\left\{\begin{array}{ll}
0, & \Delta V_{o}>0 \\
1, & \Delta V_{o}<0
\end{array}=\left\{\begin{array}{ll}
0, & C_{R E F}>C_{S E N S} \\
1, & C_{R E F}<C_{S E N S}
\end{array} .\right.\right.
$$

Based on $V_{C M P}$, the SAR logic changes the PCA digital input to increase or decrease $C_{R E F}$, using a binary search successive approximation algorithm. The logic was implemented off-chip for testing flexibility. At the end of the conversion cycle, the value of $C_{R E F}$ is matched to $C_{S E N S}$ within an error of $1 L S B$ of the PCA. The conversion time is $N \times T_{C L K}$, where $N$ is the number of bits of the PCA and $T_{C L K}$ is the period of the conversion clock $(C L K)$. The value of $V_{R E F}$ is set to $1.45 \mathrm{~V}$ which is roughly at the middle of the commonmode input-range of both the op-amp and the comparator. However, the circuit operation is insensitive to the value of $V_{R E F}$. Any variation or drift in $V_{R E F}$ will equally affect both $85 U 1$ and $U 2$, and thus it will be canceled and (4) will remain valid. The digital output of the comparator is determined by the sign of $\Delta V_{o}$ regardless of its absolute value. Any drift or variation in $V_{R E F}$ will change the absolute value of $\Delta V_{o}$ but it will not change its sign, because the sign is determined by the difference $\left(C_{R E F}-C_{S E N S}\right)$. Thus, variation in $V_{R E F}$ will not affect the circuit functionality because it will not change the output of the comparator. 


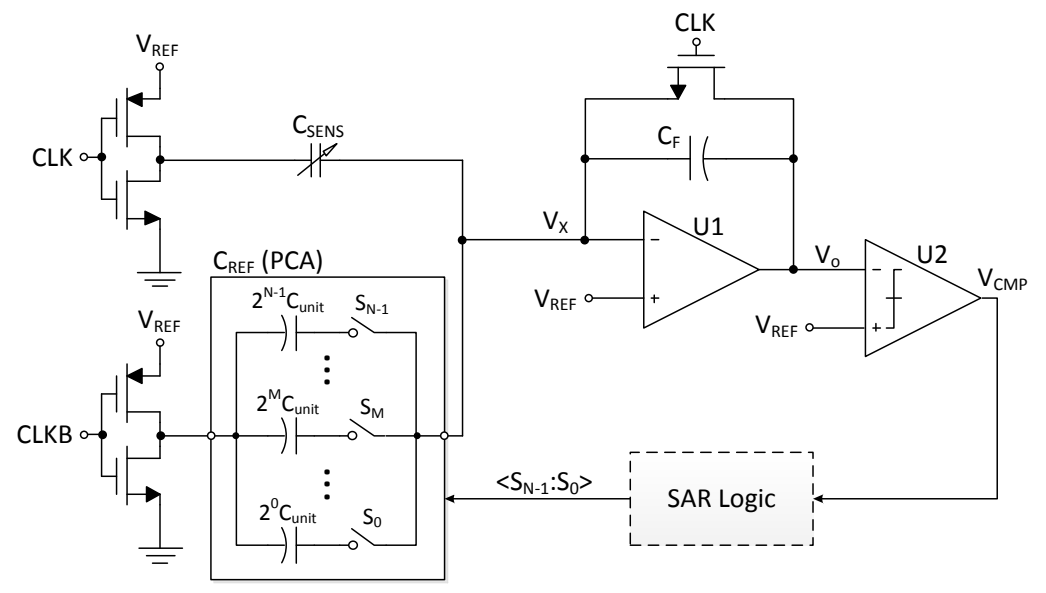

Figure 1: Schematic of the proposed SAR CDC circuit.

For a SAR ADC, only one active block (a comparator) is required, as the input signal is a buffered analog voltage. However, for a SAR CDC which directly converts capacitance to digital output, an op-amp is necessary due to several reasons. First, the capacitive sensor $\left(C_{S E N S}\right)$ is associated with large parasitic capacitors, thus it needs to be placed between two low impedance nodes, which is achieved by virtue of the op-amp virtual ground. The parasitic capacitors at the terminals of $C_{S E N S}$ and $C_{R E F}$ are either connected to ground, $V_{R E F}$, or the virtual ground inverting input of $U 1$, which are all low-impedance nodes. Thus, the parasitic capacitors do not affect circuit operation. Second, the op-amp acts as a charge amplifier, which improves the absolute resolution of the interface (LSB). Without using the op-amp (i.e., op-amp-less architecture), during the evaluate phase the charge will be redistributed between all the capacitances because the output node $\left(V_{o}=V_{X}\right)$ is a floating node. Thus, $\Delta V_{o}$ will be inversely proportional to the sum of all the capacitances connected to node $V_{x}$, i.e., $\Delta V_{o} \propto \frac{\Delta C}{C_{S E N S}+C_{R E F}+C_{P X}}$, where $C_{P X}$ is the parasitic capacitor at node $V_{X}$, instead of being inversely proportional to the small feedback capacitor $\left(C_{F}\right)$, where the sum $\left(C_{S E N S}+C_{R E F}+C_{P X}\right)$ is larger than $C_{F}$ by orders of magnitude. Moreover, thanks to the charge amplifier, the output code will not be affected by offset voltage and charge injection as will be shown in the next subsection. Third, by virtue of $U 1$ virtual ground, node $V_{X}$ which is connected to the off-chip sensor is a low impedance node, thus it will not be prone to noise coupling. 


\subsection{Circuit Nonidealities}

The previous analysis assumed ideal operation. In this section, we study the nonidealities of the op-amp, the comparator, and the feedback switch. First, considering the offset voltage of the op-amp $\left(V_{o s, o p-a m p}\right)$, in the precharge phase the charge on $C_{S E N S}, C_{R E F}$, and $C_{F}$ will be given by

$$
\begin{aligned}
q_{C_{S E N S}} & =C_{S E N S} \times\left(V_{R E F}+V_{o s, o p-a m p}\right) \\
q_{C_{R E F}} & =C_{R E F} \times V_{o s, o p-a m p} \\
q_{C_{F}} & =0
\end{aligned}
$$

Next, in the evaluate phase, the charge will be given by

$$
\begin{aligned}
q_{C_{S E N S}} & =C_{S E N S} \times V_{o s, o p-a m p} \\
q_{C_{R E F}} & =C_{R E F} \times\left(V_{R E F}+V_{o s, o p-a m p}\right) \\
q_{C_{F}} & =C_{F} \times\left(V_{R E F}+V_{o s, o p-a m p}-V_{o}\right)
\end{aligned}
$$

As the total charge remains the same in both phases, from (5) and (6), the op-amp output will be given by

$$
V_{o}=V_{R E F}+V_{R E F} \times\left(\frac{C_{R E F}-C_{S E N S}}{C_{F}}\right)+V_{o s, o p-a m p}
$$

which means that the op-amp offset voltage appears at the output as is without being amplified. By considering the comparator offset voltage $\left(V_{o s, c m p}\right)$, the comparator digital output will be given by

$$
V_{C M P}= \begin{cases}0, & \Delta V_{o}+V_{o s, o p-a m p}+V_{o s, c m p}>0 \\ 1, & \Delta V_{o}+V_{o s, o p-a m p}+V_{o s, c m p}<0\end{cases}
$$

where $\Delta V_{o}$ is given by

$$
\Delta V_{o}=V_{R E F} \times\left(\frac{C_{R E F}-C_{S E N S}}{C_{F}}\right)
$$


Moreover, due to the feedback switch, charge is injected to $C_{F}$ when the circuit moves from the precharge phase to the evaluate phase, i.e., at the falling edge of $C L K$. Thus, (8) will be modified to be

$$
V_{C M P}= \begin{cases}0, & \Delta V_{o}+V_{e r r}>0 \\ 1, & \Delta V_{o}+V_{e r r}<0\end{cases}
$$

where $V_{\text {err }}$ is the total error voltage given by

$$
V_{e r r}=V_{o s, t o t}+V_{i n j}
$$

where $V_{o s, t o t}=V_{o s, o p-a m p}+V_{o s, c m p}$ is the total offset voltage and $V_{i n j}$ is the error voltage due to switch charge injection. $V_{i n j}$ is a function of the feedback capacitance $\left(C_{F}\right)$ and increases as $C_{F}$ decreases.

The offset voltages of the op-amp and the comparator are two independent random variables. Statistical Monte Carlo simulations were used to generate their distribution, where the effects of both mismatch and process variations are captured. Fig. (2) shows the distribution of the offset voltages resulting from 500 Monte Carlo runs, where $V_{o s, o p-a m p}$ has a mean $\left(\mu_{o p-a m p}\right)$ of $0.46 \mathrm{mV}$ and a standard deviation $\left(\sigma_{o p-a m p}\right)$ of $2.06 \mathrm{mV}$, while $V_{o s, c m p}$ has a mean $\left(\mu_{c m p}\right)$ of $3.22 \mathrm{mV}$ and a standard deviation $\left(\sigma_{c m p}\right)$ of $4.70 \mathrm{mV}$. The total offset voltage $\left(V_{o s, t o t}\right)$ resulting from the sum of the two random variables will have a mean which is equal to [15]

$$
\mu_{t o t}=\mu_{o p-a m p}+\mu_{c m p}=3.68 m V
$$

and the standard deviation will be given by [15]

$$
\sigma_{\text {tot }}=\sqrt{\sigma_{o p-a m p}^{2}+\sigma_{c m p}^{2}}=5.13 \mathrm{mV}
$$

For a confidence range of $99.73 \%$, the maximum offset voltage $\left(V_{o s, \max }\right)$ is given by considering three multiples of the standard deviation, i.e., three-sigma, and thus it will be given 140 by

$$
V_{o s, \max }=\mu_{t o t}+3 \times \sigma_{t o t}=19.07 \mathrm{mV}
$$




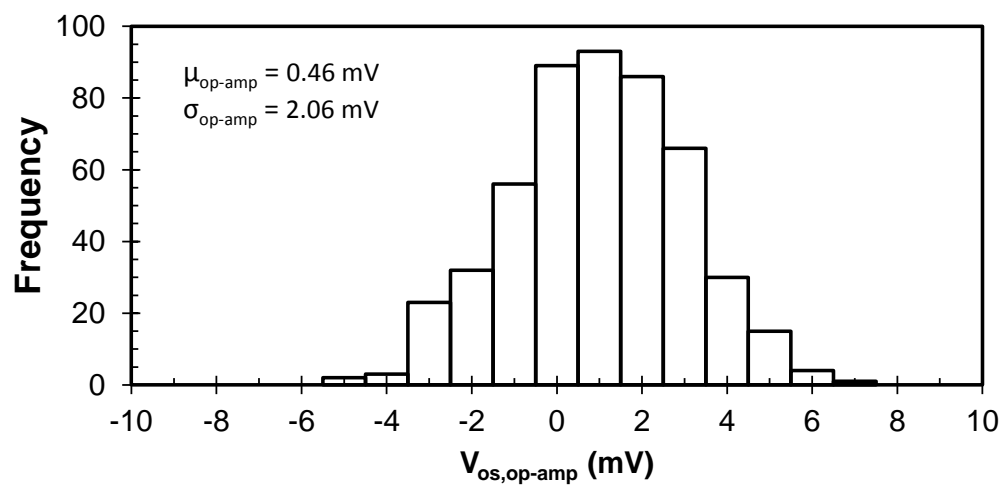

(a)

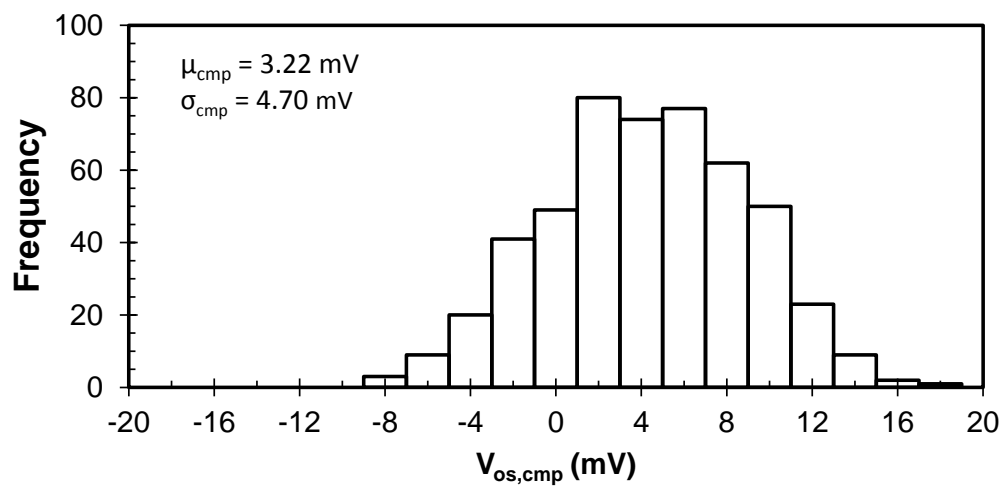

(b)

Figure 2: Offset voltage Monte Carlo simulation (500 runs) for the (a) op-amp $\left(V_{o s, o p-a m p}\right)$ and (b) comparator $\left(V_{o s, c m p}\right)$.

On the other hand, for (10) to yield the correct result, the minimum $\Delta V_{o}$ must overcome the worst case error voltage. $\Delta V_{o}$ is minimum for a minimum capacitance difference $\left(C_{R E F}-C_{S E N S}\right)$ equal to $1 L S B$ of the PCA, which is $2.75 f F$ for the implemented prototype. Thus, the condition for proper operation is given by

$$
\Delta V_{o, \min }=V_{R E F} \times \frac{L S B}{C_{F}}>V_{e r r}
$$

${ }_{145} C_{F}$ is the design parameter used to satisfy (15). But it should be noted that both $\Delta V_{o, \min }$ and $V_{e r r}$ depend on $C_{F}$. As $C_{F}$ increases, $V_{i n j}$ will decrease, but in the same time the gain will decrease, i.e., $\Delta V_{o, \min }$ will decrease. The variation of both $\Delta V_{o, \min }$ and $V_{\text {err }}$ versus $C_{F}$ for $V_{R E F}=1.45 \mathrm{~V}$ and $L S B=2.75 \mathrm{fF}$ is depicted in Fig. (3), where $V_{\text {err }}$ is given by (11) and $V_{i n j}$ is measured using transient simulation parametric sweep. The maximum 
$C_{F}$ that satisfies (15) is $131 \mathrm{fF}$. In order to account for process variations, $C_{F}$ was set to be $100 \mathrm{fF}$. A smaller value of $C_{F}$ will not be advantageous as it will negatively impact the performance. The SAR CDC can be used with finer capacitance resolution by using offset cancellation and calibration techniques, in order to reduce the effect of op-amp and comparator offsets.

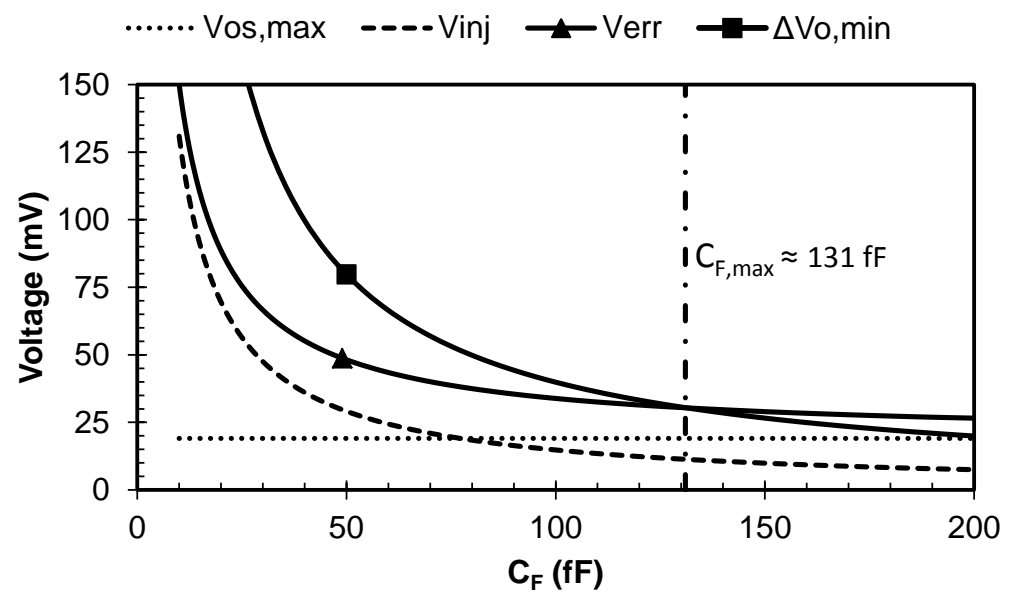

Figure 3: Variation of minimum change in output voltage $\left(\Delta V_{o, \min }\right)$ and error voltage $\left(V_{\text {err }}\right)$ versus the feedback capacitance $\left(C_{F}\right)$.

\section{Programmable Capacitor Array (PCA)}

\subsection{Mismatch Analysis}

The proposed successive approximation architecture significantly depends on the matching of the PCA. Two types of mismatch exist; systematic mismatch and random mismatch. Systematic mismatch results from process gradients and layout nonidealities. By using common centroid techniques and careful layout, the systematic mismatch can be minimized. However, random mismatch due to fabrication random variations will set the limit for achievable PCA matching. By using Pelgrom model [16], the standard deviation of the capacitor mismatch is given by

$$
\sigma\left(\frac{\Delta C_{\text {unit }}}{C_{\text {unit }}}\right)=\frac{A_{C}}{\sqrt{A_{\text {unit }}}}
$$


where $A_{C}$ is a process dependent matching parameter describing area dependence, $C_{\text {unit }}$ 165 is the unit capacitor of the array, and $A_{\text {unit }}=W \times L$ is the area of the unit capacitor, where $W$ and $L$ are the width and the length, respectively.

For a binary weighted array, the worst case mismatch occurs at mid-scale transition $(011 \cdots 1 \rightarrow 100 \cdots 0)$, because in this case all the capacitors in the array are switched. The standard deviation of the worst case DNL is given by [17]

$$
\sigma_{D N L}=\sqrt{2^{N}-1} \times \sigma\left(\frac{\Delta C_{\text {unit }}}{C_{\text {unit }}}\right) \times \frac{1}{\sqrt{2}} \approx 2^{\frac{N-1}{2}} \times \frac{A_{C}}{\sqrt{A_{\text {unit }}}}
$$

where $N$ is the number of bits. The factor of $1 / \sqrt{2}$ arises because the mismatch is given as the absolute deviation between two unit capacitors, while the DNL is calculated as the deviation from the mean value of the unit capacitor [17]. For a yield of $99.73 \%$, the threesigma of the DNL must be less than the required DNL specification $\left(D N L_{\text {spec }}\right)$ [17]

$$
3 \sigma_{D N L}<D N L_{\text {spec }}
$$

A monotonic response requires the maximum $\mathrm{DNL}$ to be less than $1 L S B$, but a more strict DNL spec of $0.5 L S B$ is used in the proposed design. Substituting with (17) yields

$$
3 \times 2^{\frac{N-1}{2}} \times \frac{A_{C}}{\sqrt{A_{\text {unit }}}}<D N L_{\text {spec }}
$$

This equation relates the number of bits of the array, the unit capacitor area, and the required DNL specification. For the given process, the matching parameter of poly-insulatorpoly (PIP) capacitors is $0.45 \%-\mu m$. Given the fixed width of the contact between poly-2 and metal-1 layers and the minimum poly-2 enclosure of the contact, the minimum PIP capacitor will have an area of $1.6 \mu \mathrm{m} \times 1.6 \mu \mathrm{m}=2.56 \mu \mathrm{m}^{2}$, which yields a unit capacitor $\left(C_{\text {unit }}\right)$, i.e., resolution, of $2.75 \mathrm{fF}$. Substituting this in (19) gives a maximum of 12.5 - bits for a $0.5 L S B$ DNL spec, which means a dynamic range of $16 \mathrm{pF}$. The previous analysis was further verified using Monte Carlo simulations. Fig. 4 shows histogram of the DNL at mid-scale transition for 12 - bit PCA using 500 Monte Carlo runs. The three-sigma of 
the DNL is $0.387 L S B$, while the DNL spec expected from (19) is $0.382 L S B$, which shows good agreement.

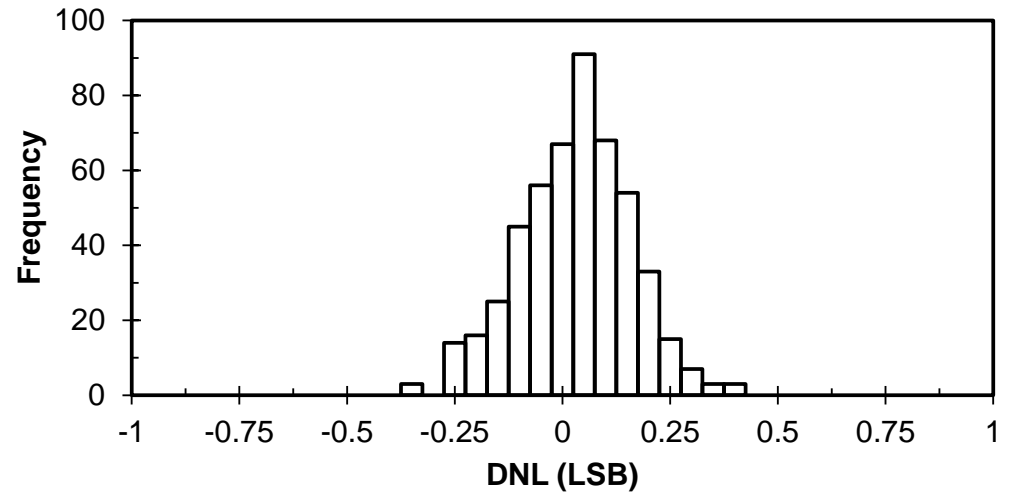

Figure 4: Histogram of DNL at mid-scale transition for 12 - bit PCA using minimum sized PIP capacitor. The histogram is generated using 500 Monte Carlo runs.

The previous limitation on PCA resolution is due to the use of standard capacitors which are provided in the technology design kit. These capacitors are optimized for area efficiency by providing high capacitance density, i.e., large capacitance in small area. By using custom designed capacitor elements, e.g., fringing capacitance between metal wires in the same layer or parasitic capacitance between two metal plates in two different layers, a much finer resolution can be achieved in a larger area, i.e., with better matching and consequently larger number of bits.

\subsection{PCA Implementation}

As previously mentioned, poly-insulator-poly (PIP) capacitors were used to implement the PCA, with a minimum unit capacitor of $2.75 \mathrm{fF}$. Due to layout design rules and routing overhead, covering a $16 \mathrm{pF}$ range with this small unit capacitor will require a very large area $\left(\approx 0.32 \mathrm{~mm}^{2}\right)$, in addition to increased complexity. Thus, a coarse-fine approach was used. A 9 - bit fine PCA with $2.75 f F$ LSB is combined with a 4 - bit coarse PCA with $1 p F$ LSB. The full scale of the fine PCA is $\approx 1.4 p F$, such that it covers the step size of the coarse PCA under all process variations, and thus a continuous range is ensured. This 
approach resulted in a total area $\approx 0.05 \mathrm{~mm}^{2}$, which means an area reduction of $84 \%$ compared to the former one.

A common centroid layout was used for both PCAs to reduce the effect of mismatch due to process gradients [18]. Fig. 5 illustrates the proposed layout plan for the fine PCA. A conventional common centroid layout will involve large systematic mismatch due to routing to the LSBs at the center of the PCA. Thus, the array was split into two mirrored halves with a channel in between for routing and switches, where each half is surrounded with dummy capacitors. Metal-3 was used for routing, while Metal-2 was used to shield the PCA to avoid any systematic mismatch.

\begin{tabular}{|c|c|c|c|c|c|c|c|c|c|c|c|c|c|c|c|c|c|c|c|c|c|c|c|c|c|c|c|c|c|c|c|c|}
\hline & & & & & & & & & & & & & & & & & & & & & & & & $D$ & $\mathrm{D}$ & $D$ & D & D & & D & & \\
\hline & & & & & & & & & & & & & & & & & & & & & & & & & & & & & & & & \\
\hline & & $\sqrt{C}$ & & & & & & & & & & & & & & & & & & & & & & & & & & & & & & \\
\hline & & \begin{tabular}{l|l}
88 & $C$ \\
\end{tabular} & & & & & & & & & & & & & & & & & & & & & & & & & & & & & & \\
\hline & & \begin{tabular}{l|l|l}
88 &
\end{tabular} & & $\mathrm{C7}$ & & & & & & & & & & & & & & & & & & & & & 6] & $\mathrm{Cl}$ & & & & & & \\
\hline & & $c 8$ & & $C 7$ & & & & & & & & & & & & & & & & & & & & & & & & & & & & \\
\hline & C8 & $\begin{array}{c}88 \\
\end{array}$ & & C8 & & & & cc & & & & & $\mathrm{C4}$ & & & & & & & & & & & & & & & & & & & \\
\hline & & $=8$ & & & & & & & & & & & & 8 & & & & & $\mathrm{C8}$ & & & & & & & & & & & & & \\
\hline & & & & & & & & & & & & & & 88 & col & & & $\mathrm{C} 1$ & & & & & & & & & & & & C8 & & \\
\hline & c & 88 & & $C 8$ & & & & & & & & & & & & & & $\mathrm{D}$ & & & & & & & & & & 28 & & & & \\
\hline & c & & & & & & & & & & & & & C8 & & & & & & & & & & & & & & & & & & \\
\hline & & $8 \mathrm{C}$ & & & & $C^{2}$ & & C & & & & & $\mathrm{C4}$ & & & & & & $\mathrm{C3}$ & & & & & & & & & & & & & \\
\hline & $8 C$ & & & $C$ & & & & & & & & & & $\mathrm{C4}$ & & & & $\mathrm{C} 4$ & & & & & & & & & & & & & & \\
\hline & & & & & & & & & & & & & & & & & & & & & & & & & & & & & & & & \\
\hline & & & & & & & & & & & & & & & & & & & & & & & & & & & & & & & & \\
\hline & & & & & & & & C & & & & & & & & & & & & & & & & & & & & & & & & \\
\hline & & & & & & $C \varepsilon$ & $C$ & C8 & $C \varepsilon$ & & & & & & & & & & & & & & $\mathrm{C} 8$ & $8 \mid C 8$ & & & & & C8 & $\mathrm{C} 8$ & & \\
\hline & & & & & & & & & & & & & & & & & & & & & & & & & & & & & & & & \\
\hline
\end{tabular}

Figure 5: Split common centroid layout scheme of the 9 - bit fine PCA with a routing channel in between the two halves. 'D' indicates a dummy unit capacitor.

\section{System Description and Digital Interface}

The block diagram of the proposed system is shown in Fig. 6. At the heart of the system is the SAR CDC circuit, which is connected to two capacitors: a capacitive sensor $\left(C_{S E N S}\right)$ and a reference capacitor $\left(C_{R E F}\right)$. The reference capacitor terminals is connected to the reference PCA and can be connected to an external reference capacitor $\left(C_{R E F_{-} E X T}\right)$, where the connection is controlled by a digital signal. The reference PCA is used in performing the SAR algorithm, while $C_{R E F_{-} E X T}$ is an optional external reference capacitance, which can be used in case the sensor baseline capacitance is larger than the range of the reference PCA. Similarly, the sensing capacitor can be connected to an external capacitive 
sensor $\left(C_{S E N S_{-} E X T}\right)$ or an on-chip dummy sensor, which is included to be used in the testing process.

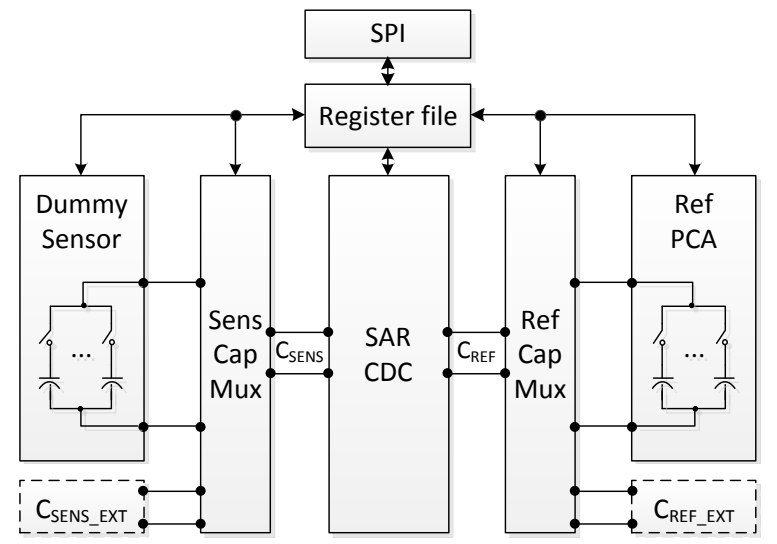

Figure 6: Block diagram of the proposed CDC system.

All digital signals for control, PCA read/write, etc, are managed by a register file, which can be accessed through a 4-wire serial peripheral interface (SPI) bus. Fig. (7) shows a schematic for the digital interface block diagram. The slave SPI interface was used in order to simplify the testing process and minimize the bonding pads and package pins. The slave SPI interface is composed of four signals: 1) serial clock (SCLK) generated by the master controlling the bus , 2) master output slave input (MOSI), 3) master input slave output (MISO), and 4) active low slave select (SSB).

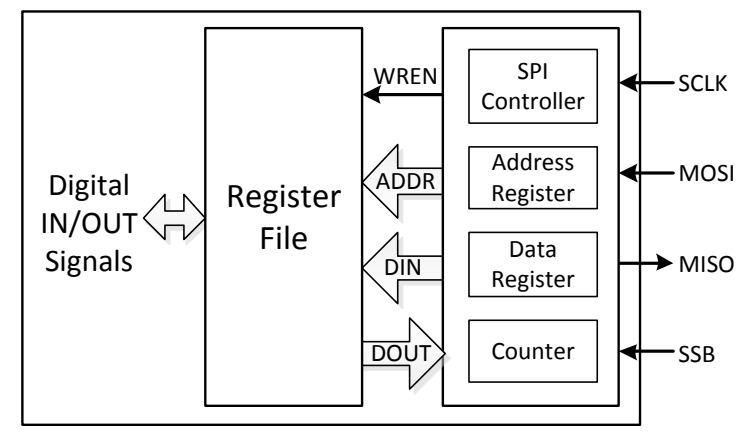

Figure 7: Block diagram of the digital interface.

The designed SPI works for Mode '0', which means that the idle value of SCLK is zero, data is sampled with the SCLK rising edge, and generated with SCLK falling edge. There is no standard format for the SPI frame. For the implemented design, the first bit was used 
as RD/WRB control bit, followed by seven address bits and multiples of eight data bits. Frame structure and timing diagrams of generic read and write transactions are shown in Fig. 8. The SPI controller generates signals to read and write the register file. The address register is automatically incremented after each data byte, which enables reading/writing multiple registers in the same transaction. The SPI controller state machine solely uses the externally provided clock (SCLK), which eliminates the need for sampling the digital inputs at a higher frequency rate and maintains low power consumption.

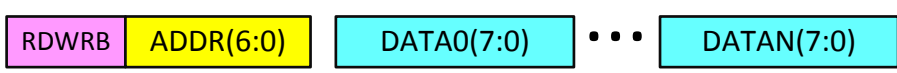

(a)

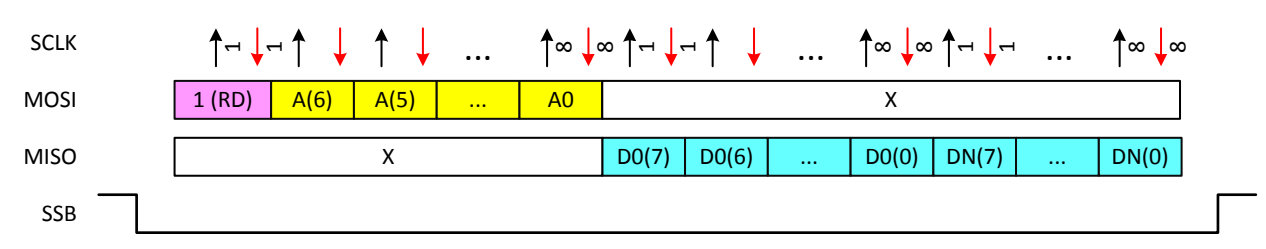

(b)

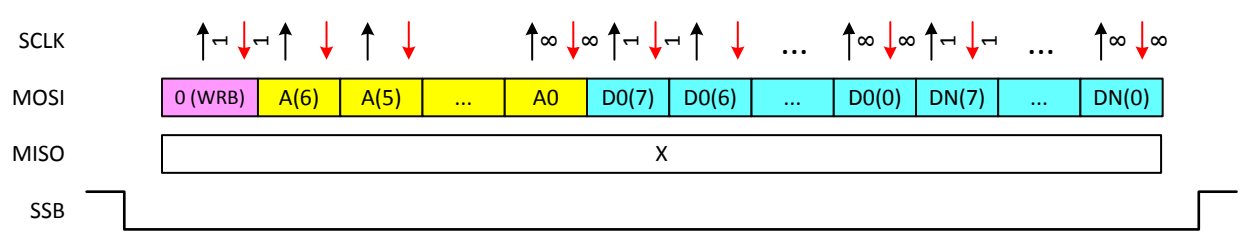

(c)

Figure 8: SPI interface (a) frame structure and timing diagram of (b) read transaction and (c) write transaction.

\section{Experimental Results} interface is shown in Fig. 10. Communication with the chip SPI interface was done using 
an Atmel AT90USB1287 USB powered microcontroller and the SPI bus was monitored by Intronix LA1034 Logic Analyzer.

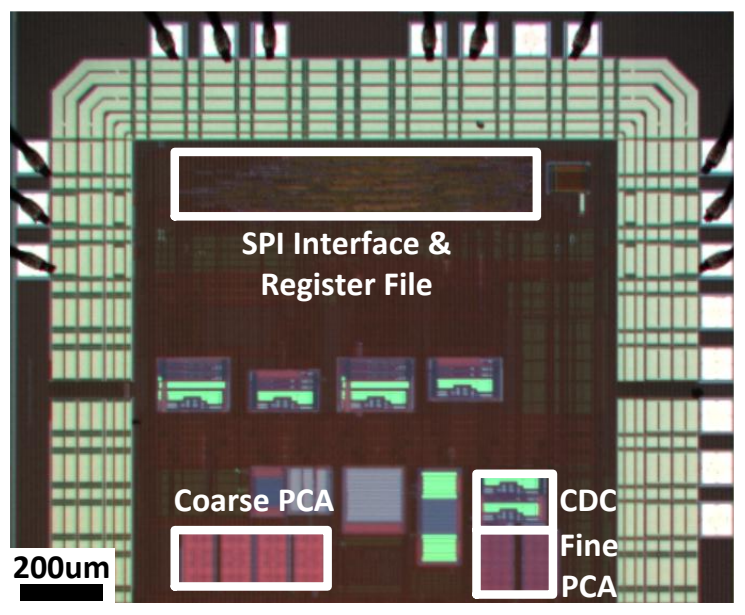

Figure 9: Die photograph. Blocks used in the proposed design are labeled.

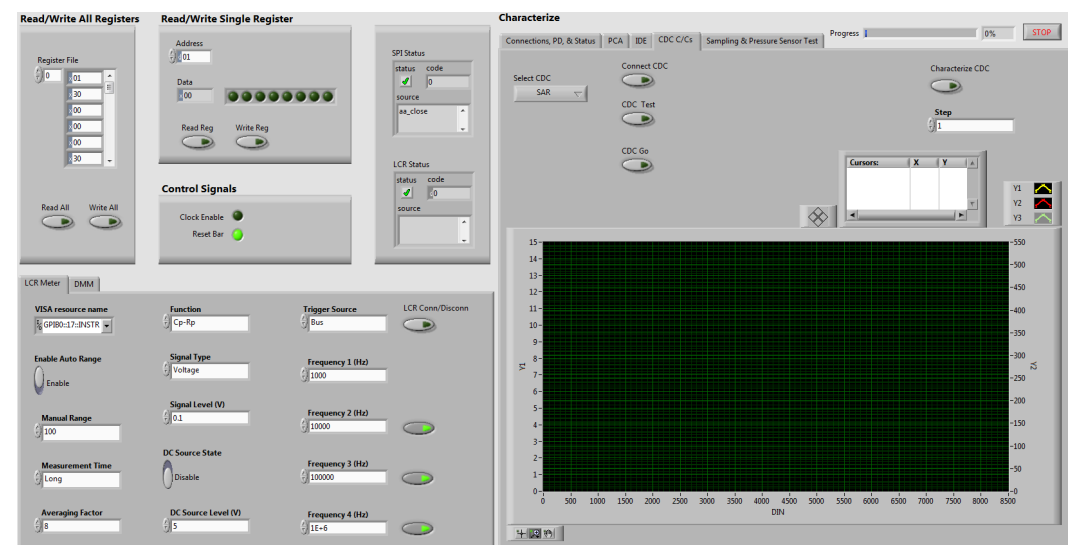

Figure 10: Snapshot of the LabVIEW interface used for test equipment control and data acquisition. circuit over a limited part of its dynamic range. 
In order to test the fabricated prototype both on-chip dummy sensor and off-chip capacitive sensor were used. A digitally programmable dummy capacitive sensor is included onchip to simplify the testing process. Fig. 11 shows the measured conversion result of the SAR CDC versus the dummy sensor capacitance. The capacitance of the dummy sensor was measured using Agilent E4980A LCR meter. The measured characteristics in Fig. 11 shows the linear relation between the output code and the input capacitance over the interface circuit dynamic range.

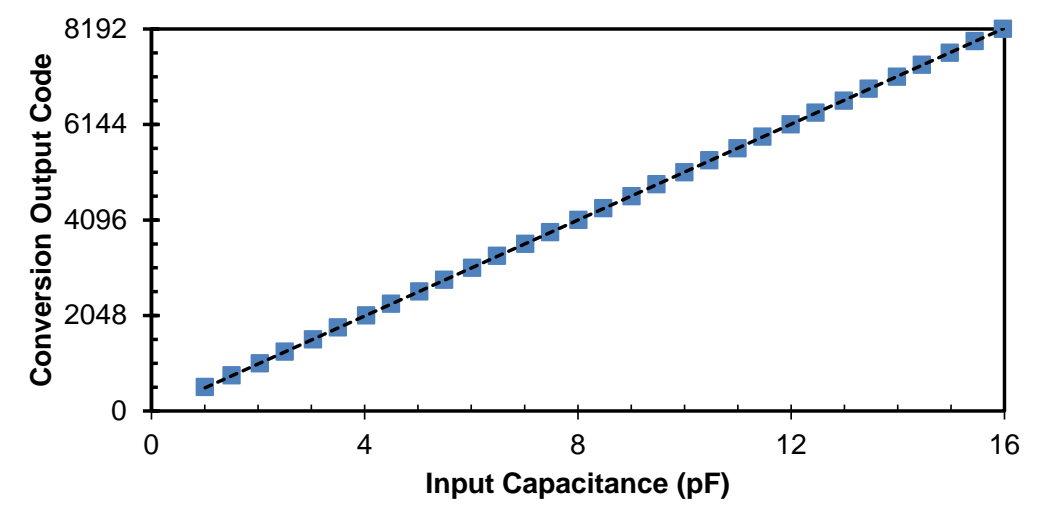

Figure 11: Transfer characteristics of the CDC showing the measured output code versus the capacitance of the dummy sensor.

To further verify the operation and the robustness of the proposed circuit, an external MEMS capacitive pressure sensor from Protron Mikrotechnik was used [20]. A schematic of the test setup used in pressure measurement is shown in Fig. 12. A custom test cell is designed, equipped with three FNPT ports for gas inlet, gas outlet, and vacuum, in addition to four hermetic BNC connectors. Two pressure sensors are placed inside the test cell: Proton capacitive pressure sensor and MPX4250A, which is a voltage output pressure sensor from Freescale. The SAR CDC test board is connected to the capacitive sensor inside the test cell using coaxial test leads. The voltage output of MPX4250A is digitized using Agilent 43301A Multimeter. Both the capacitance reading by the SAR CDC test board and the pressure reading of MPX4250A are recorded by a LabVIEW program. 


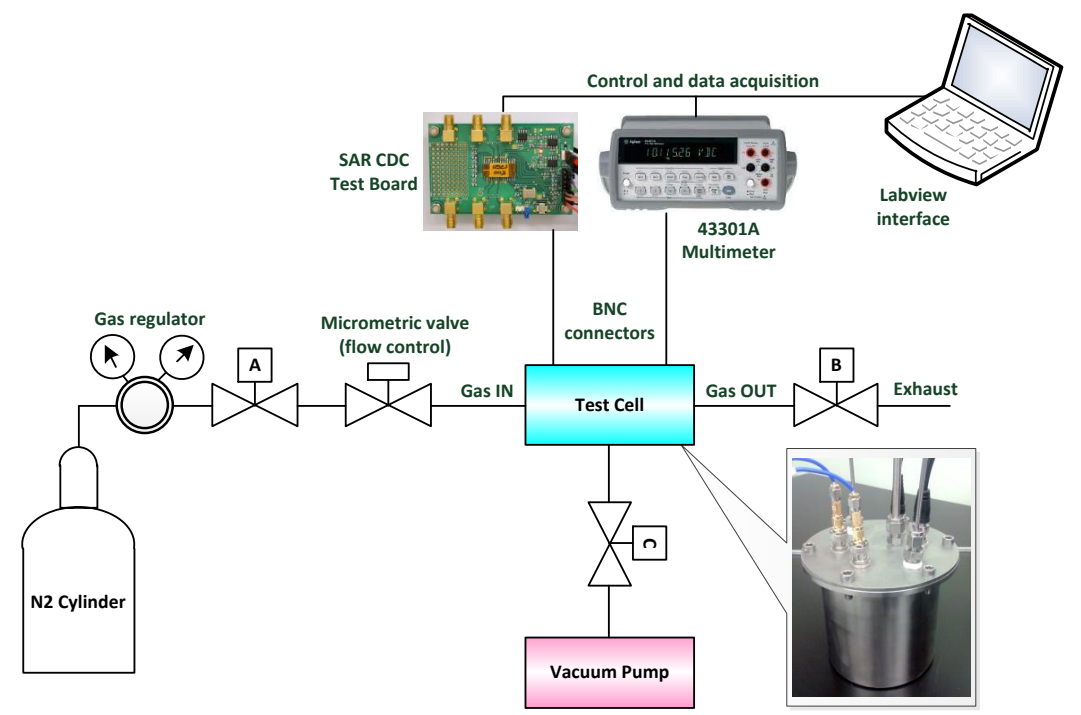

Figure 12: Schematic of the test setup used in pressure measurement and a photograph of the custom hermetic test cell.

The Proton MEMS capacitive pressure sensor is operated in both its barometric pressure mode and high pressure mode. The capacitive pressure sensor has nonlinear characteristics as shown by the dotted line in Fig. 13 [20]. The measured SAR CDC conversion result follows the expected nonlinear characteristics of the sensor. It should be noted that in this measurement setup, large parasitic capacitances exist at the terminals of $C_{S E N S}$ due to the test cell, connectors, and cabling. Thus, this test verifies the robustness of the proposed design in the presence of large parasitic capacitance. 
- SAR CDC Measurement - - Sensor characteristics

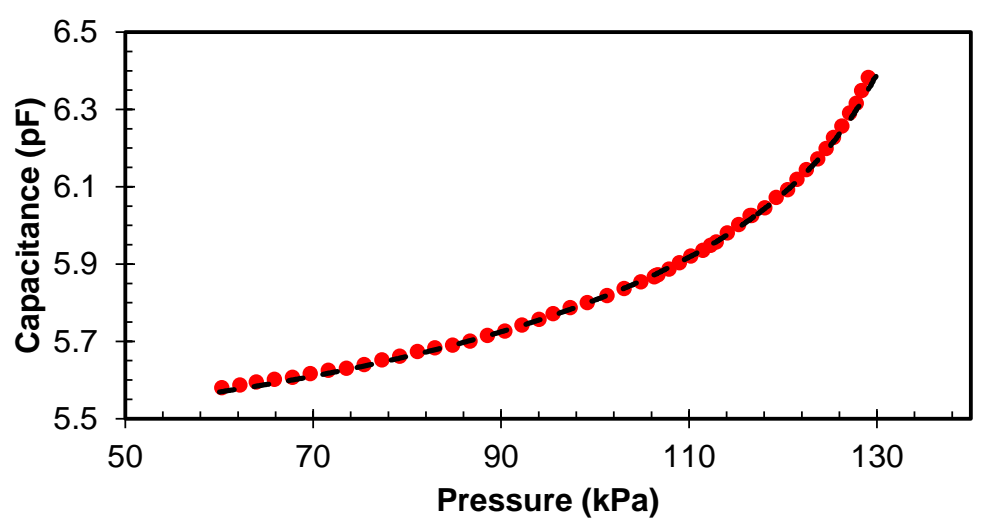

(a)

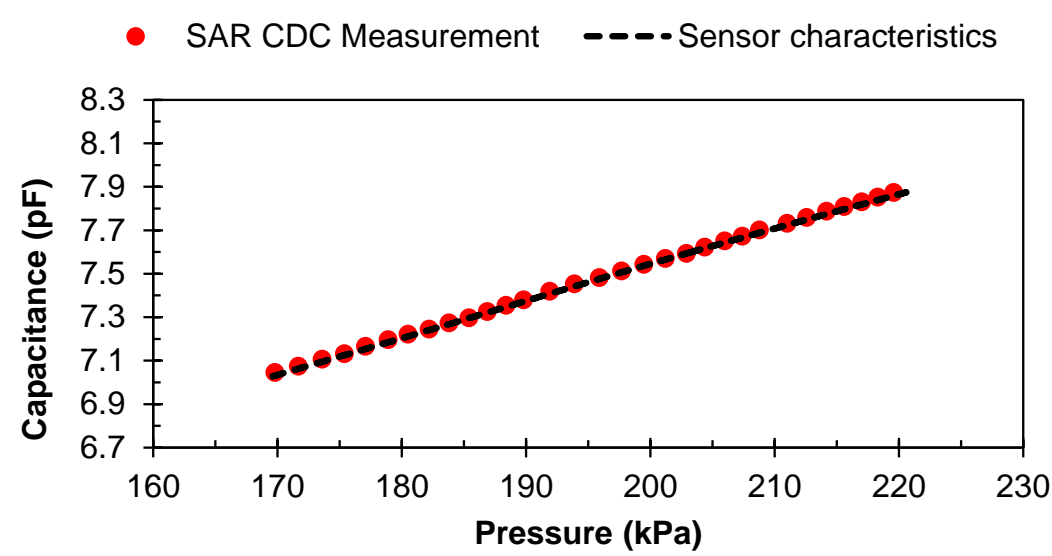

(b)

Figure 13: Capacitance measurement using SAR CDC overlaid on MEMS pressure sensor nonlinear characteristics. (a) Sensor operated in its barometric pressure mode. (b) Sensor operated in its high pressure mode.

In order to determine the resolution of the interface, the method described in [12] was used, where the standard deviation of the output is measured for a fixed input capacitance applied to the CDC. Measurements show that the output code is repeatable with variation of 1 LSB only, which shows that the output is quantization-noise limited [21], i.e., limited by the smallest unit capacitor in the PCA.

Fig. 14 shows a complete SAR CDC conversion cycle captured using an Agilent DSOX2024A oscilloscope. The snapshot shows the clock signal $(C L K)$, the buffered op-amp output $\left(V_{o}\right)$, and the comparator digital output $\left(V_{C M P}\right)$, for an output code that has alternating zeros and ones (OxOAAA) for the purpose of illustration. As seen in the figure, $V_{o}$ approaches 
$V_{R E F}=1.45 \mathrm{~V}$ as the capacitance of the reference PCA $\left(C_{R E F}\right)$ is successively approximated to $C_{S E N S}$, based on $V_{C M P}$. The period of the clock signal $(C L K)$ is $75 \mu s$ in order to allow the microcontroller to sample $V_{C M P}$ and serially communicate with the chip using the SPI interface. However, for on-chip logic, the clock period is limited by $V_{o}$ settling time when clock is high $(C L K=1)$ and the maximum delay between the clock falling edge and the comparison result $\left(V_{C M P}\right)$, which result in a minimum clock period of $50 \mu \mathrm{s}$. This translates to a total conversion time equal to $650 \mu \mathrm{s}$.

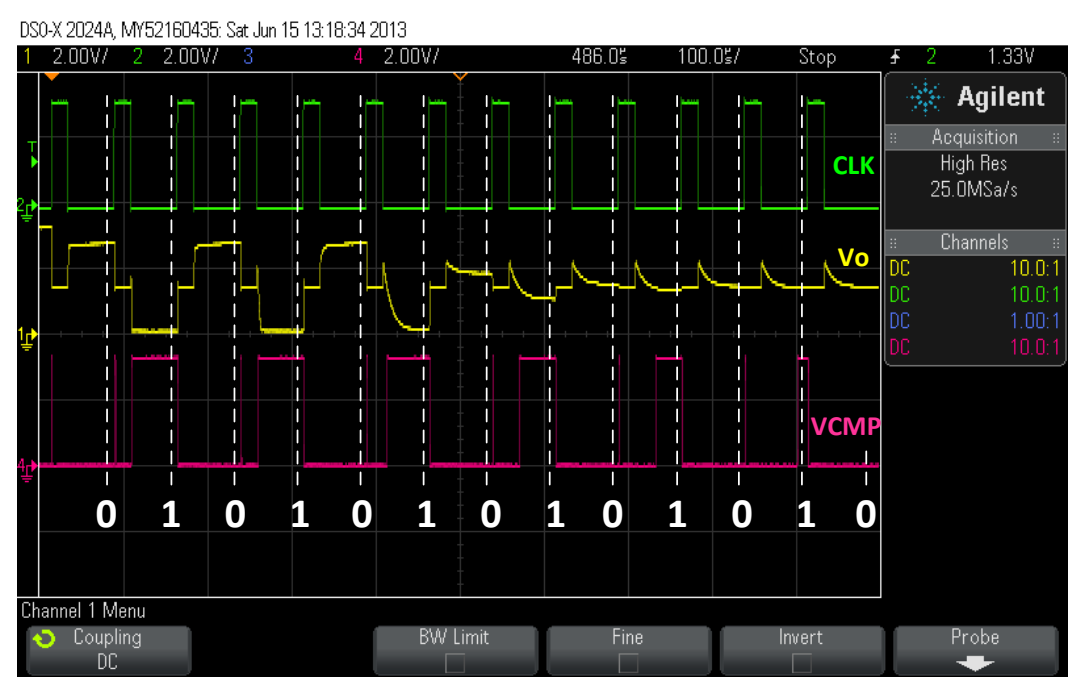

Figure 14: Scope snapshot showing a complete SAR CDC conversion cycle for an output code of OxOAAA.

The power consumption of the SAR CDC circuit depends on the sensing capacitance $\left(C_{S E N S}\right)$, being minimum at $C_{S E N S}=0$ and maximum at full-scale, i.e., $C_{S E N S}=16 p F$. Table 1 summarizes the average current drawn by each block calculated using post-layout transient simulations and the experimentally measured total current consumption. Most of the power is consumed by the op-amp $(\approx 94 \%)$. The total power consumption estimated from simulation is close to the actual value measured in the lab. The measured current consumption ranges from $65 \mu \mathrm{A}$ to $92 \mu \mathrm{A}$, which translates to a power consumption range between $214 \mu W$ and $303 \mu W$ for a $3.3 \mathrm{~V}$ supply. 
Table 1: Average current drawn by each block calculated using post-layout simulation and experimentally measured total current consumption.

\begin{tabular}{|c|c|c|c|}
\hline & & $\begin{array}{l}\text { Post-layout } \\
\text { simulation }\end{array}$ & $\begin{array}{c}\text { Experimentally } \\
\text { measured }\end{array}$ \\
\hline \multirow{4}{*}{$C_{S E N S}=0$} & Op-amp & $62.4 \mu A(94.4 \%)$ & - \\
\hline & Comparator & $3.49 \mu A(5.3 \%)$ & - \\
\hline & Reference voltage $\left(V_{R E F}\right)$ & $0.19 \mu A(0.3 \%)$ & - \\
\hline & Total & $66.1 \mu \mathrm{A}$ & $65 \mu A$ \\
\hline \multirow{4}{*}{$C_{S E N S}=16 p F$} & Op-amp & $80.7 \mu A(93.9 \%)$ & - \\
\hline & Comparator & $5.01 \mu A(5.8 \%)$ & - \\
\hline & Reference voltage $\left(V_{R E F}\right)$ & $0.26 \mu A(0.3 \%)$ & - \\
\hline & Total & $85.9 \mu \mathrm{A}$ & $92 \mu A$ \\
\hline
\end{tabular}

The proposed circuit is insensitive to the absolute value of any analog voltage or current, which means it has inherently very low temperature sensitivity. The only source of deviation with temperature is the temperature coefficient of the PIP capacitors that form the PCA, which is one or two decades less than temperature coefficients of resistors, commonly used in capacitance-to-time converters $[9,22]$. Fig. 15 shows the measured capacitance over the complete range of the CDC at two different temperatures, namely $25^{\circ} \mathrm{C}$ and $65^{\circ} \mathrm{C}$, where the two graphs almost coincide. The zoomed-in inset shows that the deviation is only $0.1 \%$ of full-scale range (FSR) for a temperature difference of $40^{\circ} \mathrm{C}$, which means a temperature coefficient of $25 \mathrm{ppm} /{ }^{\circ} \mathrm{C}$. This value is more than one decade better than the temperature insensitive design reported in [9].

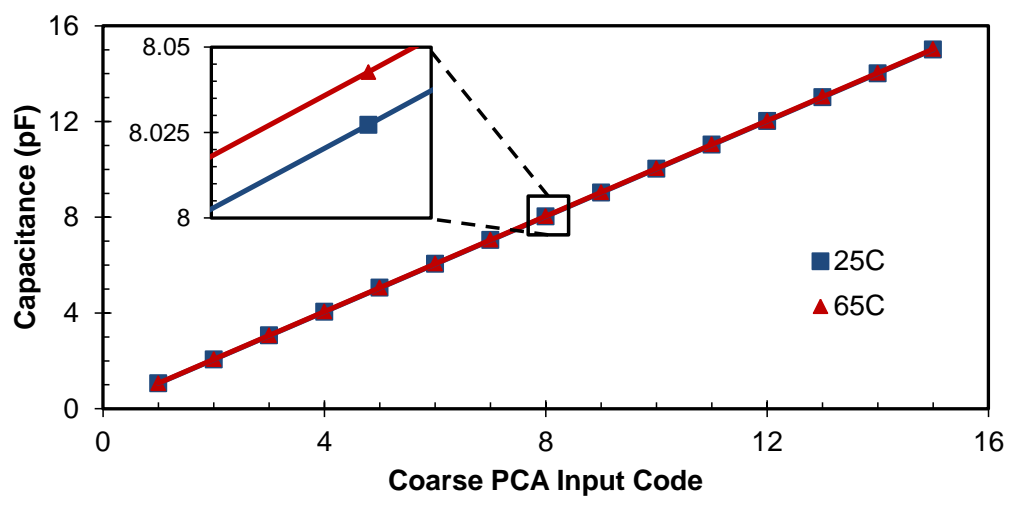

Figure 15: Measured capacitance of the coarse PCA at $25^{\circ} \mathrm{C}$ and $65^{\circ} \mathrm{C}$ vs coarse PCA input code. The zoomed-in inset shows the deviation to be only $0.1 \%$ of FSR. 
In order to evaluate the energy efficiency of the proposed design, the energy efficiency figure-of-merit (FoM) widely used to compare ADCs and CDCs is used. The FoM is given by

$$
F_{o M}=\frac{P_{a v g} \times T_{c o n v}}{2^{R}}
$$

where $P_{\text {avg }}$ is the average power consumption, $T_{\text {conv }}$ is the conversion time, and $R$ is the resolution in bits. Substituting with the worst power consumption of $303 \mu \mathrm{W}$, a conversion time of $650 \mu \mathrm{s}$ and 12.5 -bit resolution yields a FoM of $34 \mathrm{pJ} / \mathrm{step}$, which is the best reported to date for a CDC that generates a direct digital output.

A summary for the performance parameters of the proposed prototype compared with state-of-the-art CDCs is given in Table 2. Both conversion time and power consumption affect energy efficiency, thus the offline calibration range of [11] is not considered, as it is done once during initialization phase, and is not included in the reported conversion time and power consumption. It should be noted that capacitance-to-time converters reported in $[9,12]$ generate a semi-digital output that needs further digitization using a time-to-digital converter, e.g., a counter running at a high frequency clock, which is not considered in the reported power consumption. Similarly, for $\Delta \Sigma$ architectures in [10,11,23, 24] the output is in the form of a bit stream that needs to be filtered by a digital decimation filter, which is again not considered in the reported power consumption. In spite of that, the proposed design achieves better energy efficiency compared to designs using the same technology and supply voltage $[9,12,10,11]$. The designs in $[23,24]$ report better energy efficiency, however they use a different supply voltage which makes a fair comparison difficult. Noting that in $[23,24]$ the power consumption of the digital decimation filter is not included, in addition to the different supply voltage, the proposed design achieves comparable energy efficiency.

\section{Conclusion}

We proposed a robust energy-efficient SAR CDC that achieves an energy-efficiency FoM of $34 \mathrm{pJ} / \mathrm{step}$, which is better than state-of-the-art CDCs that are implemented using the same 
Table 2: Performance summary and comparison.

\begin{tabular}{|c|c|c|c|c|c|c|c|c|}
\hline & $\begin{array}{l}\text { JSSC } \\
2007 \text { [9] }\end{array}$ & $\begin{array}{l}\text { JSSC } \\
2012 \text { [12] }\end{array}$ & $\begin{array}{l}\text { AD7153 } \\
{[13]}\end{array}$ & $\begin{array}{l}\text { ESSCIRC } \\
2008 \text { [23] }\end{array}$ & $\begin{array}{l}\text { A-SSCC } \\
2011[24]\end{array}$ & $\begin{array}{l}\text { TCAS-II } \\
2011[10]\end{array}$ & $\begin{array}{l}\text { ISSCC } \\
2012[11]\end{array}$ & $\begin{array}{l}\text { This } \\
\text { Work }\end{array}$ \\
\hline Architecture & \multicolumn{2}{|c|}{ Capacitance-to-time } & \multicolumn{5}{|c|}{$\Delta \Sigma$} & SAR \\
\hline Output format & PWM & PM & $\begin{array}{l}\text { Digital } \\
\text { code }\end{array}$ & $\begin{array}{l}\text { Bit } \\
\text { stream }\end{array}$ & $\begin{array}{l}\text { Bit } \\
\text { stream }\end{array}$ & $\begin{array}{l}\text { Bit } \\
\text { stream }\end{array}$ & $\begin{array}{l}\text { Bit } \\
\text { stream }\end{array}$ & $\begin{array}{l}\text { Digital } \\
\text { code }\end{array}$ \\
\hline Technology $(\mu m)$ & 0.35 & 0.35 & NA & 0.35 & 0.16 & 0.35 & 0.35 & 0.35 \\
\hline Area $\left(m m^{2}\right)$ & 0.2 & 0.51 & NA & 1 & 0.25 & 0.048 & 2.6 & 0.07 \\
\hline Power $(m W)$ & 16.5 & 0.211 & 0.33 & 0.83 & 0.01 & 1.44 & 15 & 0.303 \\
\hline $\begin{array}{l}\text { Supply Voltage } \\
(V)\end{array}$ & 3.3 & 3.3 & 3.3 & 1.8 & 1.8 & 3.3 & 3.3 & 3.3 \\
\hline $\begin{array}{l}\text { Conversion Time } \\
(m s)\end{array}$ & 0.05 & 7.6 & 5 & 0.025 & 10.2 & 0.128 & 0.02 & 0.65 \\
\hline $\begin{array}{l}\text { Capacitance } \\
\text { Range }^{a}(p F)\end{array}$ & 0.4 & 6.8 & 4 & NA & 0.8 & 1 & 0.1 & 16 \\
\hline $\begin{array}{l}\text { Absolute } \\
\text { Resolution }(f F)\end{array}$ & 0.9 & 0.2 & 1 & NA & 0.1 & 0.49 & 0.065 & 2.75 \\
\hline Resolution $^{b}$ (bit) & 8.8 & 15 & 12 & 10.2 & 13 & 11 & 10.6 & 12.5 \\
\hline FoM $(p J / s t e p)$ & 1851 & 49 & 403 & 18 & 13 & 90 & $193^{c}$ & 34 \\
\hline Notes & $\begin{array}{l}\text { Power cons } \\
\text { digital conve }\end{array}$ & $\begin{array}{l}\text { tion of time-to- } \\
\text { is not reported }\end{array}$ & & Power & $\begin{array}{l}\text { onsumption of } \\
\text { ilter is not repor }\end{array}$ & ital decimation & & \\
\hline
\end{tabular}

* $\mathrm{PM}=$ Period modulation, $\mathrm{PWM}=$ Pulse width modulation, $\mathrm{NA}=$ Not available .

${ }^{a}$ Capacitance Range $=C_{\max }-C_{\min }$. Offline calibration range is not considered.

${ }^{b}$ Resolution $=\log _{2}\left(\frac{\text { Capacitance Range }}{\text { Absolute Resolution }}\right)$.

${ }^{c}$ Calculated based on the reported capacitance range and absolute resolution. Offline calibration range that is not included in conversion time is not considered.

supply voltage. The proposed CDC provides a direct digital output without the need of digital filtering or time-to-digital conversion. The use of charge amplifier stage enables robust operation against parasitic capacitances, offset voltages, charge injection, and noise coupling. Measurement results show that the proposed SAR CDC is an attractive solution for applications that require wide capacitance range, compact area, and energy efficient operation. The operation of the CDC was experimentally verified over its complete $16 p F$ capacitance range. Measurement results for testing the proposed CDC with MEMS pressure sensor were provided. The proposed design has low temperature sensitivity of $25 \mathrm{ppm} /{ }^{\circ} \mathrm{C}$ as it does not depend on analog references. The operating principle is independent of supply voltage, and thus by scaling down the supply voltage and the feature size, a significant improvement in both power consumption and resolution can be achieved, leading to even better FoM. 


\section{References}

355 [1] L. K. Baxter, Capacitive sensors design and applications, IEEE Press, 1997.

[2] T. Pedersen, G. Fragiacomo, O. Hansen, E. Thomsen, Highly sensitive micromachined capacitive pressure sensor with reduced hysteresis and low parasitic capacitance, Sensors and Actuators A: Physical 154 (1) (2009) 35 - 41. doi:http://dx.doi.org/10.1016/j.sna.2009.07.013.

[3] E. Jafer, C. S. Ibala, Design and development of multi-node based wireless system for efficient measuring of resistive and capacitive sensors, Sensors and Actuators A: Physical 189 (0) (2013) 276 - 287. doi:http://dx.doi.org/10.1016/j.sna.2012.09.023.

[4] Z. Rittersma, Recent achievements in miniaturised humidity sensors-a review of transduction techniques, Sensors and Actuators A: Physical 96 (23) (2002) 196 210. doi:10.1016/S0924-4247(01)00788-9.

[5] H. Muhammad, C. Oddo, L. Beccai, C. Recchiuto, C. Anthony, M. Adams, M. Carrozza, D. Hukins, M. Ward, Development of a bioinspired \{MEMS $\}$ based capacitive tactile sensor for a robotic finger, Sensors and Actuators A: Physical 165 (2) (2011) 221 - 229. doi:http://dx.doi.org/10.1016/j.sna.2010.10.025.

[6] V. Tsouti, C. Boutopoulos, I. Zergioti, S. Chatzandroulis, Capacitive microsystems for biological sensing, Biosensors and Bioelectronics 27 (1) (2011) 1 - 11. doi:10.1016/j.bios.2011.05.047.

[7] A. Kummer, A. Hierlemann, Configurable electrodes for capacitive-type sensors and chemical sensors, Sensors Journal, IEEE 6 (1) (2006) 3 - 10. doi:10.1109/JSEN.2005.856547.

[8] P. J. Mazzone, Exhaled breath volatile organic compound biomarkers in lung cancer, Journal of Breath Research 6 (2) (2012) 027106.

[9] P. Bruschi, N. Nizza, M. Piotto, A current-mode, dual slope, integrated capacitanceto-pulse duration converter, Solid-State Circuits, IEEE Journal of 42 (9) (2007) 1884 -1891. doi:10.1109/JSSC.2007.903102. 
[10] D.-Y. Shin, H. Lee, S. Kim, A delta-sigma interface circuit for capacitive sensors with an automatically calibrated zero point, Circuits and Systems II: Express Briefs, IEEE Transactions on 58 (2) (2011) 90-94. doi:10.1109/TCSII.2010.2104015.

[11] S. Xia, K. Makinwa, S. Nihtianov, A capacitance-to-digital converter for displacement sensing with $17 \mathrm{~b}$ resolution and 20 us conversion time, in: Solid-State Circuits Conference Digest of Technical Papers (ISSCC), 2012 IEEE International, 2012, pp. 198200. doi:10.1109/ISSCC.2012.6176973.

[12] Z. Tan, S. Shalmany, G. C. M. Meijer, M. A. P. Pertijs, An energy-efficient 15-bit capacitive-sensor interface based on period modulation, Solid-State Circuits, IEEE Journal of 47 (7) (2012) 1703-1711. doi:10.1109/JSSC.2012.2191212.

[13] Analog Devices, AD7153 Datasheet. URL http: //www.analog.com

[14] K. Tanaka, Y. Kuramochi, T. Kurashina, K. Okada, A. Matsuzawa, A 0.026mm2 capacitance-to-digital converter for biotelemetry applications using a charge redistribution technique, in: Solid-State Circuits Conference, 2007. ASSCC '07. IEEE Asian, 2007, pp. 244 -247. doi:10.1109/ASSCC.2007.4425776.

[15] C. M. Grinstead, J. L. Snell, Introduction to probability, American Mathematical Soc., 1998.

[16] M. Pelgrom, A. C. J. Duinmaijer, A. Welbers, Matching properties of mos transistors, Solid-State Circuits, IEEE Journal of 24 (5) (1989) 1433-1439. doi:10.1109/JSSC.1989.572629.

[17] P. Harpe, C. Zhou, Y. Bi, N. van der Meijs, X. Wang, K. Philips, G. Dolmans, H. De Groot, A 26 uW 8 bit 10 MS/s Asynchronous SAR ADC for Low Energy Radios, Solid-State Circuits, IEEE Journal of 46 (7) (2011) 1585-1595. doi:10.1109/JSSC.2011.2143870.

[18] A. Hastings, The art of analog layout, 2nd Edition, Prentice Hall, 2006. 
[19] W. Bracke, P. Merken, R. Puers, C. V. Hoof, Generic architectures and design methods for autonomous sensors, Sensors and Actuators A: Physical 135 (2) (2007) 881 - 888. doi:http://dx.doi.org/10.1016/j.sna.2006.07.028.

410

[20] Protron Mikrotechnik GmbH, Micro-Machined Capacitive Absolute Pressure Sensors Datasheet (Jan 2012).

URL http://www.protron.de

[21] W. Bracke, P. Merken, R. Puers, C. Van Hoof, Ultra-low-power interface chip for autonomous capacitive sensor systems, Circuits and Systems I: Regular Papers, IEEE Transactions on 54 (1) (2007) 130-140. doi:10.1109/TCSI.2006.887978.

[22] F. Reverter, O. Casas, Direct interface circuit for capacitive humidity sensors, Sensors and Actuators A: Physical 143 (2) (2008) 315 - 322. doi:http://dx.doi.org/10.1016/j.sna.2007.11.018.

[23] S. A. Jawed, D. Cattin, M. Gottardi, N. Massari, A. Baschirotto, A. Simoni, A 828 UW 1.8V 80dB dynamic-range readout interface for a MEMS capacitive microphone, in: Solid-State Circuits Conference, 2008. ESSCIRC 2008. 34th European, 2008, pp. 442-445. doi:10.1109/ESSCIRC.2008.4681887.

[24] Z. Tan, R. Daamen, A. Humbert, K. Souri, Y. Chae, Y. Ponomarev, M. A. P. Pertijs, A 1.8v 11uw cmos smart humidity sensor for rfid sensing applications, in: Solid State Circuits Conference (A-SSCC), 2011 IEEE Asian, 2011, pp. 105-108. doi:10.1109/ASSCC.2011.6123615. 